\title{
Sequence Spaces and Stability of Integer Translates
}

\author{
QTYU SuN
}

In this paper, we introduce sequence spaces of Triebel-Lizorkin type, especially Hardy spaces and bounded mean oscillation spaces. We establish $F_{p}^{\alpha q}$-stability of integer translates of general distributions which extends the results of [7.8] to general distributions. Also the corresponding David-Journe theorem for sequences is established.

Key words: Spaces of Triebel-Lizorkin type, stability of integer translates, David-Journé theorom, sampling theorems, global linear independence, D-transforms, multiresolution analysis

AMS subject classification: $41 \mathrm{~A} 63,46 \mathrm{~A} 45,42 \mathrm{~B} 20$

\section{Introduction}

The main objective of this paper is to study stability of integer translates, sequence spaces of Triebel-Lizorkin type and the David-Journé theorem for sequences. The $l^{2}$-stability of integer translates of univariate spline functions, exponential spline functions, cardinal spline functions, etc. has well studied in last ten years or more early. Ron [11] gave a criterion for the $l^{2}$-stability of integer translates of a compactly supported distribution by global linear independence. The $l^{p}$-stability $(1<p<\infty)$ of integer translates of a function which satisfies some two-scale difference equation was studied by Jia, Micchelli and Wang $[7,8]$. In this paper, the $l^{p}$-stability of integer translates of any compactly supported distribution is considered. The $l^{p}$-spaces are classical sequence spaces. In this paper, we use the Littlewood-Paley theory for sequences [4, Chapter 7] to define sequence spaces $F_{p}^{\alpha q}(Z)$ of Triebel-Lizorkin type on $Z$ (the set of all integers) and establish two connections between $F_{p}^{\alpha q}(Z)$ and $F_{p}^{\alpha q}(R)$, the function spaces of Triebel-Lizorkin type on $R$ (the set of all reals). We construct first an immersion operator from $F_{p}^{\alpha q}(Z)$ to $F_{p}^{\alpha q}(R)$. Secondly we find appropriate function $\phi$ such that, for $f \in S^{\prime}(Z)$ (the set of all tempered sequences), the conclusions $f=\{f(n)\} \in F_{p}^{\alpha q}(Z)$ and $\sum_{n} f(n) \phi(x-n) \in F_{p}^{\alpha q}(R)$ are equivalent. For example, as such function $\phi$ there can appear univariate spline function $B_{k}$ with degree $k \geq \max (\alpha+3,2)$ and a generating function $\phi$ with high regularity of a multiresolution analysis of $L^{2}(R)$. The $l^{2}$-boundedness of infinite matrices is subject with a long history going back to Hardy, Littlewood and Paley, and to Schur and Hilbert (see [9]). The David-Journé theorem for sequences is devoted to some class of infinite matrices which is of Calderon-Zygmund type.

Q. Sun: Zhojiang University, Center Math. Sci., Hangzhou, Zhojiang 310027, and Hangzhou University Dop. Math., Hangzhou, Zhejiang 310028, P. R. of China. This project is partially supported by Postdoctoral Followship Foundation of China and Zhojiang Provincial Natural Science Foundation of China. 
The inspiration comes from two aspects directly. One comes from multiresolution analysis introduced by Meyer and Mallat. We know that the $l^{P}$-stablity of integer translates of the generating function $\phi \in L^{p}(1 \leq p<\infty)$, i.e.

$$
C^{-1}\|a\|_{l^{p}} \leq\left\|\sum_{n} a(n) \phi(x-n)\right\|_{L^{p}} \leq C\|a\|_{\text {lP }}
$$

for all $a=\{a(n)\} \in l^{p}$ and some constant $C$ independent of sequences $a$, plays an essential role in multiresolution analysis [7]. Hereafter we denote by $\|\cdot\|_{L^{p}}$ the norm of p-integrable functions and by $\|\cdot\|_{i p}$ the norm of p-summable sequences. The other one comes from sampling theory. It is well known that the $L^{p}$-norm of a function $f \in L^{p}(R)$ with support of its Fourier transform $\hat{f}$ contained in $(-\pi, \pi)$ is comparable to the $l^{p}$-norm of its sampling values on the integer lattices $f(n)(n \in Z)$ where $1<p<\infty$ (see $[6,13])$. In particular; we can write $f(x)=\sum_{n \in Z} f(n) \phi(x-n)$ for some appropriate function $\phi$, which is essentially $l^{p}$-stability of integer translates of $\phi$.

The theory of function spaces is well-studied in last thirty years [14]. In recent years, Frazier and Jawerth unificd the function spaces of Triebel-Lizorkin-Besov type by using $\dot{\phi}$-transform (see [5] and references therein). Our problem to consider is which appropriate sequence spaces should be introduced when $L^{p}$ in (1) is replaced by Hardy spaces or more generally by spaces of Triebel-Lizorkin-Besov type and under which appropriate conditions on $\phi(1)$ holds.

Similar to the sequence spaces of Besov type introduced by Torres [13] and the sampling theorem for sequences [6], we define the sequence spaces of Triebel-Lizorkin type in Section 2 by using Littlewood-Paley theory [4, Chapter 7] and study some fundamental properties. Especially we define the Hardy space $H^{1}(Z)$ and bounded mean oscillation space $B M O(Z)$ and give their characterizations.

The David-Journe theorem on $R$ is an important contribution to the development of harmonic analysis [3]. We establish a corresponding theorem for sequences in Section 3 .

The stability of integer translates arised in the interpolation of sequences by functions. The $l^{p}$-stability of integer translates of the generating function $\phi$ of a multiresolution analysis is well-studied. Cohen, Jia, Sun and Wang etc. gave some characterization to $l^{2}$. stability by using zero point sets of the characteristic trigonometric series $H$ and eigenvalues of the characteristic polynomial $P$, respectively (see $[1,8]$ and references therein). Jia and Wang $[8]$ pointed out that the $l^{p}$-stability $(1 \leq p \leq \infty)$ is equivalent to each other in one spatial dimension. Let $\phi$ be a compactly supported distribution. We say that the integer translates of $\phi$ are globally linearly independent for tempered sequences if, for all $a=\{a(n)\}$ so that $|a(n)| \leq C(1+|n|)^{N}$ for some $C, N>0$, the conclusion $\sum_{n \in Z} a(n) \phi(x-n)=0$ implies $a(\bar{n})=0$ for all $n \in Z$. Let $\left\{\phi_{j}\right\}_{j=1}^{l}$ be a family of compactly supported distributions. We say that the integer translates of $\left\{\phi_{j}\right\}_{j=1}^{\prime}$ are globally linearly independent for tempered sequences if the integer translates of $\sum_{j=1}^{l} a_{j} \phi_{j}$ are globally linearly independent for tempered sequences for every $\sum_{j=1}^{l}\left|a_{j}\right|^{2} \neq 0$, or

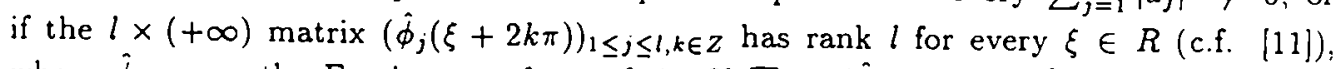
where $\hat{\phi}$ means the Fourier transform of $\phi$. If $\sum_{k \in Z}\left|\hat{\phi}_{j}(\xi+2 k \pi)\right|^{2}<+\infty$ for every $\xi \in R$, then the integer translates of $\left\{\phi_{j}\right\}_{j=1}^{\prime}$ are globally linearly independent for tempered 
sequences if and only if $\left(a_{i j}(\xi)\right)_{1 \leq i, j \leq l}$ are uniformly positive definite matrices, where $a_{i j}(\xi)=\sum_{k \in Z} \hat{\phi}_{i}(\xi+2 k \pi) \hat{\hat{\phi}}_{j}(\xi+2 k \pi)$. In [7], Jia and Micchelli pointed out that the $l^{p}$-stability of integer translates of $\phi$ are equivalent to each other when $\phi$ satisfies some two-scale difference equation in high spatial dimensions. In Section 4 , we consider the inequality

$$
C^{-1} \sum_{j=1}^{l}\left\|a_{j}\right\|_{l p} \leq\left\|\sum_{j=1}^{l} \sum_{n \in Z} a_{j}(n) \phi_{j}(x-n)\right\|_{L,} \leq C \sum_{j=1}^{l}\left\|a_{j}\right\|_{l p}
$$

where $a_{j}=\left\{a_{j}(n)\right\}_{n \in Z} \in l^{p}$. More generally the corresponding inequalities on spaces of Triebel-Lizorkin type are considered. We show (Theorem 11 and Corollary) that the left inequality of $\left(1^{\prime}\right)$ holds if and only if the integer translates of $\left\{\phi_{j}\right\}_{j=1}^{\prime}$ are globally linearly independent for tempered sequences, which extends the result of Jia and Micchelli [7] to general distributions. But we encounter difficulty to treat the right inequality of $\left(1^{\prime}\right)$ on general spaces of Triebel-Lizorkin type (Theorem 8), though we get a simple characterization on the Hardy space $H^{1}(Z)$ (Theorem 13). Also we give a method to study sequence spaces via function spaces. Precisely we use Theorem 9 to get a characterization of the Hardy space $H^{1}(Z)$ and bounded mean oscillation space $B M O(Z)$ by the Hilbert transform.

For simplicity in the exposition we restrict ourselves to the one dimensional case, all the results can be easily extended to high spatial dimensions.

The author would like to thank the referee for his useful suggestions and careful corrections. Also thanks to Dr. J. Synnatzschke to his suggestion on the representation of the material and correction in English.

\section{Sequence spaces of Triebel-Lizorkin type}

We begin the study of sequence spaces of Triebel-Lizorkin type with a decomposition of Littlewood-Paley type, or $\phi$-transform. To this end, we introduce the concept of an admissible pair. First let

$$
\begin{aligned}
& \mathcal{S}(Z)=\left\{f=\{f(n)\}: f \text { a function on } Z,|f(n)| \leq C_{N}(1+|n|)^{-N}, C_{N}>0, \forall N>0\right\} \\
& \mathcal{S}^{\prime}(Z)=\left\{f=\{f(n)\}: f \text { a function on } Z,|f(n)| \leq C(1+|n|)^{N} \text { for some } N, C>0\right\},
\end{aligned}
$$

and

$$
\mathcal{P}(Z)=\left\{f=\{f(n)\}_{n \in Z}: \quad f \text { is a polynomial on } R\right\},
$$

the set of polynomial sequences. For a function $\Phi$ on $R$ and $f$ on $Z$ the Fourier transforms of $\Phi$ is defined by $\hat{\Phi}$ and the Fourier series of the sequences $f=\{f(n)\}$ is defined by $\hat{f}(x)=\sum_{n \in Z} f(n) e^{i n x}(x \in R)$. Let $Z_{-}=\{v \in Z: v \leq 0\}$ and for $v \in Z_{-}$

$$
T_{v}=\left\{\begin{array}{ll}
{[-\pi, \pi] \backslash\left[-\frac{3}{8} \pi, \frac{3}{8} \pi\right]} & \text { for } v=0 \\
{\left[-3 \cdot 2^{v-2} \pi, 3 \cdot 2^{v-2} \pi\right] \backslash\left[-3 \cdot 2^{v-3} \pi, 3 \cdot 2^{v-3} \pi\right]} & \text { for } v \leq-1
\end{array} .\right.
$$


Definition 1. We say that $\left(\varphi_{v}, \psi_{v}\right) \in S(Z) \times S(Z)$ is an admissible pair on $Z$ if

$$
\begin{aligned}
& \operatorname{supp} \hat{\varphi}_{\nu}, \operatorname{supp} \hat{\psi}_{\nu} \subset\left[-2^{\nu} \pi, 2^{v} \pi\right] \backslash\left[-2^{\nu-2} \pi, 2^{\nu-2} \pi\right] \\
& \sum_{\nu=-\infty}^{0} \overline{\hat{\varphi}}_{\nu}(x) \hat{\psi}_{\nu}(x)=1 \quad \text { for } \quad x \in[-\pi, \pi] \backslash\{0\}
\end{aligned}
$$

and if there exist constants $A$ and $A_{k}(k \geq 0)$, independent of $v$, such that

$$
\begin{array}{ll}
\left|\left(\frac{\partial}{\partial x}\right)^{k} \dot{\varphi}_{v}(x)\right|,\left|\left(\frac{\partial}{\partial x}\right)^{k} \hat{\psi}_{v}(x)\right| \leq A_{k} 2^{-k v} & \text { for } x \in[-\pi, \pi] \text { and }-k, v \leq 0 \\
\left|\hat{\varphi}_{v}(x)\right|_{v}\left|\hat{\psi}_{v}(x)\right| \geq A & \text { for } x \in T_{v} .
\end{array}
$$

For an admissible pair $\left(\varphi_{v}, \psi_{v}\right) \in S(Z) \times S(Z)$ on $Z$ we define sequences $\left\{\varphi_{v, k}\right\}_{k \in Z}$ and $\left\{\psi_{v, k}\right\}_{k \in Z}$ by

$$
\varphi_{v, k}(n)=2^{-v / 2} \varphi_{v}\left(n-2^{-v} k\right) \text { and } \psi_{v, k}(n)=2^{-v / 2} \psi_{v}\left(n-2^{-v} k\right)
$$

Lemma 1. Let $\left(\varphi_{v}, \psi_{v}\right) \in S(Z) \times S(Z)$ be an admissible pair. Then, for $f \in$ $S^{\prime}(Z) \backslash \mathcal{P}(\mathcal{Z})$, we have

$$
f=\sum_{v=-\infty}^{0} \sum_{k \in Z}\left\langle f, \varphi_{v, k}\right\rangle \psi_{v, k}
$$

where the inner product $\langle\cdot, \cdot\rangle$ is defined by $(f, g)=\sum_{n \in Z} f(n) \bar{g}(n)$.

We omit the proof of Lemma 1 since a detailed proof for $f \in l^{2}(Z)$ was given in $[6]$. The correct interpretation of $(6)$ is that, for $f \in S^{\prime}(Z)$, there exists a non-negative integer $M$ and a family $\left\{P_{N}\right\}_{N \geq 0} \subset \mathcal{P}(Z)$ with degree of $P_{N}$ at most $M$ such that

$$
f=\lim _{N \rightarrow \infty}\left(\sum_{\nu=-N}^{0} \sum_{k \in Z}\left(f, \varphi_{v, k}\right) \psi_{v, k}+P_{N}\right)+P_{0} \quad \text { in } S^{\prime}(Z)
$$

Definition 2. Let $\alpha \in R, 0<p, q \leq+\infty$ and let $\left(\phi_{v}, \psi_{v}\right) \in S(Z) \times S(Z)$ be an admissible pair on $Z$.

(i) we define the sequence spaces $F_{p}^{\alpha q}(Z)$ of Triebel-Lizorkin type as the collection of complex-valued sequences $f \in \mathcal{S}^{\prime}(Z) / \mathcal{P}(Z)$ such that the quasinorms

$$
\begin{aligned}
& \|f\|_{F_{p}^{a^{\prime}}(z)}=\left\|\left(\sum_{v \leq 0}\left(2^{v \alpha}\left|\varphi_{v} * f\right|\right)^{q}\right)^{\frac{1}{l}} \cdot\right\|_{l p} \quad(0<p<\infty) \\
& \|f\|_{F_{\infty}^{\prime \prime}(z)}=\sup _{k, v} 2^{v} \sum_{2-v k \leq n<2-v(k+1)} \sum_{0 \geq \mu \geq v}\left(2^{\mu \alpha}\left|\varphi_{\mu} * f(n)\right|^{q}\right)^{\frac{1}{q}}
\end{aligned}
$$

are finite, where $g * f(n)=\sum_{k \in Z} g(n-k) f(k)$ for $g \in S(Z)$ and $f \in S^{\prime}(Z)$, the $1^{q}$-norm is replaced by the supremum over $v$ or $\mu$ if $q=+\infty$. 
(ii) Furthermore, we define the sequence spaces $f_{p}^{\alpha q}(Z)$ as the collection of complexvalued sequences $s=\left\{s_{v, k}\right\}_{(v, k) \in Z_{-} \times Z}$ such that the quasinorms

$$
\begin{aligned}
& \|s\|_{f_{p}^{a}(Z)}=\left\|\left(\sum_{v \in Z_{-, k} \in Z}\left(2^{v \alpha}\left|s_{v, k}\right| \chi_{v, k}\right)^{q}\right)^{\frac{1}{q}}\right\|_{l p}(0<p<\infty) \\
& \|s\|_{f_{\infty}^{a q}(Z)}=\sup _{v, k}\left(2^{v} \sum_{2^{-v} v_{k \leq n<2^{-v}(k+1)}}\left(\sum_{\substack{2^{-v} \sum_{k} \leq 2^{-\mu} k^{\prime} \\
2^{-\mu}\left(k^{\prime}+1\right)<2^{-v}(k+1)}}\left(2^{\mu \alpha}\left|s_{\mu, k^{\prime}}\right| x_{\mu, k^{\prime}}(n)^{q}\right)^{\frac{1}{q}}\right)\right)
\end{aligned}
$$

are finite, where we denote

$$
\chi_{v, k}(n)= \begin{cases}2^{-v / 2} & \text { when } 2^{-v} k \leq n<2^{-v}(k+1) \\ 0 & \text { otherwise. }\end{cases}
$$

Theorem 1. Suppose that $\alpha \in R, 0<p<+\infty, 0<q \leq \infty$ and that $\left(\varphi_{v}, \psi_{v}\right) \in$ $\mathcal{S}(Z) \times \mathcal{S}(Z)$ is an admissible pair on $Z$. Then

$$
S_{\varphi}: F_{p}^{\alpha q}(Z) \ni f \longmapsto\left\{\left(f, \varphi_{v, k}\right)\right\} \in f_{p}^{\alpha q}(Z)
$$

and

$$
T_{\psi}: f_{p}^{\alpha q}(Z) \ni\left\{s_{v, k}\right\} \longmapsto \sum_{(v, k) \in Z_{-} \times Z} s_{v, k} \psi_{v, k} \in F_{p}^{\alpha q}(Z)
$$

are bounded operators. Furthermore $T_{\psi} S_{\varphi}=1$ on $F_{p}^{\alpha q}(Z)$ and $\|f\|_{F_{p}^{\alpha q}(Z)} \approx\left\|S_{\varphi} f\right\|_{f_{p}^{\alpha q}(Z)}$, i.e.,

$$
C^{-1}\|f\|_{F_{p}^{a q}(Z)} \leq\left\|S_{\varphi} f\right\|_{f_{p}^{\alpha q}(Z)} \leq C\|f\|_{F_{p}^{\circ q}(Z)} \quad\left(\forall f \in F_{p}^{\alpha q}(Z)\right)
$$

for some constant $C$ not depending of $f$.

The corresponding relation between $F_{p}^{\alpha q}(R)$ and $f_{p}^{\alpha q}(R)$ is given in [5, Theorem 2.2]. The proof of Theorem 1 can follow the proof of Theorem 2.2 and Theorem 3.2 in [5] line by line with the Fefferman-Stein vector-valued maximal inequality there being replaced by the following lemma.

Lemma 2., Suppose $1<p<+\infty$ and $1<q \leq+\infty$. Then, for any sequences $f_{i}=\left\{f_{i}(n)\right\}_{n \in Z} \in \mathbb{P}^{P}$, there exists a constant $C$ independing of $f_{i}$ such that

$$
\left\|\left(\sum_{i \in Z}\left|M f_{i}\right|^{q}\right)^{\frac{1}{q}}\right\|_{1 p} \leq C\left\|\left(\sum_{i \in Z}\left|f_{i}\right|^{q}\right)^{\frac{1}{q}}\right\|_{1 p} \text {, }
$$

where the Hardy-Littlewood maximal operator $M$ on $Z$ is defined by

$$
M f_{i}(k)=\sup _{a \leq k \leq b, a, b \in Z} \frac{1}{b-a} \sum_{a \leq n<b}\left|f_{i}(n)\right| .
$$


Lemma 2 follows from the Fefferman-Stein vector-valued maximal inequality [5, Theorem A.1) and the following observation. For a sequence $f=\{f(n)\}$, we define a function $\tilde{f}$ on $R$ by $f(x)=f(n)$ for $n \leq x<n+1$. Therefore there exists an absolute constant $C$ such that $C^{-1} \tilde{M} \tilde{f}(x) \leq M f(n) \leq C \tilde{M} \tilde{f}(x)$ for $n \leq x<n+1$, where $\tilde{M}$ denotes the Hardy-Littlewood maximal operator on $R$ as usual.

Remark 1. By Theorem 1, we know that the definition of $F_{p}^{\alpha q}(Z)$ is independent of admissible pairs.

Define a natural map $I: f_{p}^{\alpha q}(Z) \longrightarrow f_{p}^{\propto q}(R)$ by

$$
(I s)_{Q}= \begin{cases}s_{v, k} & \text { when } v \leq 0 \\ 0 & \text { when } v>0\end{cases}
$$

for $s=\left\{s_{v, k}\right\}$ where $Q=\left\{2^{-v} k, 2^{-v}(k+1)\right)$. Therefore we have

$$
C^{-1}\|s\|_{f_{p}^{\alpha q}(Z)} \leq\|I s\|_{f_{p}^{\alpha q}(R)} \leq C\|s\|_{f_{p}^{\alpha q}(Z)} \quad\left(\forall s \in f_{p}^{\alpha q}(Z)\right)
$$

for some constant $C$ independent of $s$. Using the above map we can study the dual spaces and atomic decomposition of $F_{p}^{\alpha q}(Z)$. Precisely by the dual theorem in $[5$, Section 5$]$ and Theorem 1 we have

Theorem 2. Suppose $\alpha \in R$ and $0<q<+\infty$. Let $q^{\prime}=\frac{q}{q-1}$ for $1<q<\infty$ and $q^{\prime}=+\infty$ for $0<q \leq 1$. Then

(i) $\left(F_{p}^{\alpha q}(Z)\right)^{*}=F_{p^{\prime}}^{-\alpha q^{\prime}}(Z)$ for $1 \leq p<+\infty$.

(ii) $\left(F_{p}^{\alpha q}(Z)\right)^{*}=F_{\infty}^{\beta \infty}(Z)$ for $0<p<1$, where $\beta=-\alpha+n\left(\frac{1}{p}-1\right)$.

For $\alpha \in R$ and $0<p<+\infty, 0<q \leq+\infty$ let

$$
N_{0}=\max ([1 / \min (p, q, 1)-1-\alpha]+1,-1) \text { and } K_{0}=[\alpha+2]_{+},
$$

where $[a]$ denotes the integer part of the number $a$.

Definition 3: Let $a_{v, k} \in F_{p}^{\alpha, q}(Z)$ for $(v, k) \in Z-\times Z$ and fix $\tilde{N} \geq N_{0}, \tilde{K} \geq K_{0}$. We say that $\left\{a_{v, k}\right\}_{(v, k) \in Z_{-} \times Z}$ is a family of $(\tilde{K}, \tilde{N})-$ smooth atoms of $F_{p \text {. }}^{\alpha q}(Z)$ if

(i) supp $a_{v, k} \subset 3 Q_{v, k}$

(ii) $\sum_{n \in Z} n^{\gamma} a_{v, k}(n)=0$ for $|\gamma| \leq \tilde{N}$

(iii) $\left\|\Delta^{\gamma} a_{v, k}(n)\right\|_{1 \infty} \leq 2^{v / 2+\gamma v}$ for $|\gamma| \leq \tilde{K}$, where $Q_{v, k}=\left[2^{-v} k, 2^{-v}(k+1)\right)$.

Theorem 3. Let $\alpha \in R, 0 \leq q \leq+\infty$ and $0<p<+\infty$. Then for each sequence $f \in$ $F_{p}^{\alpha q}(Z)$ there exists a family of $(\tilde{K}, \tilde{N})$-smooth atoms $\left\{a_{v, k}\right\}_{(v, k) \in Z-\times Z}$ and coefficients $s=$ $\left\{s_{v, k}\right\} \in f_{p}^{\alpha Q}(Z)$ such that $f=\sum_{v, k} s_{v, k} a_{v, k}$ in $\mathcal{S}^{\prime}(Z) \backslash \mathcal{P}(Z)$ and $\|s\|_{f_{p}^{\alpha}(Z)} \leq C\|f\|_{F_{p}^{a q}(Z)}$, 
where $C$ is some positive constant. Conversely we have $\left\|\sum_{v, k} s_{v, k} a_{v, k}\right\|_{F_{p}^{a q}(\dot{Z})} \leq C$ $\|s\|_{f_{p}^{a}(Z)}$ for any family of $(\tilde{K}, \tilde{N})$-smooth atoms $\left\{a_{v, k}\right\}$ and $s=\left\{s_{v, k}\right\}_{(v, k) \in Z_{-} \times Z}$, where $C$ is a positive constant independent of $s$.

Proof. We follow the same procedure as in [5, Theorem 4.1]. For $v \leq 0$, let $P_{v}=$ $\left\{P_{v}(n)\right\}_{n \in Z}$ be a family of sequences defined with help of Fourier series by

$$
\hat{P}_{v}(x)=\sum_{n \in Z} P_{v}(n) e^{i n x}=2^{2 v N}\left(\sin \frac{2^{-v-1}+1}{2} x / \sin \frac{x}{2}\right)^{2 M+2 N}\left(1-e^{-i x}\right)^{2 M},
$$

where $M$ and $N$ are large integers chosen later. Obviously the $\hat{P}_{v}$ are trigonometric polynomials with degree less than $(M+N) 2^{-v}+2 M,\left|\hat{P}_{v}(x)\right| \geq A$ for all $|x| \in\left[2^{v-2} \pi, 2^{v} \pi\right]$, and $\left|\left(\frac{\partial}{\partial x}\right)^{\gamma} \hat{P}_{v}(x)\right| \leq A_{\gamma} 2^{-v \gamma}$ for $\gamma \geq 0$ for some constant $A$ and $A_{\alpha}$ independent of $v \leq 0$.

Let $\left(\dot{\phi}_{v}, \psi_{v}\right) \in \mathcal{S}(Z) \times \mathcal{S}(Z)$ be an admissible pair and $\eta_{v} \in \mathcal{S}(Z)$ be defined with help of Fourier series by $\hat{\eta}_{v}=\hat{\psi}_{v} / \hat{P}_{v}$. Therefore $\hat{\eta}_{v}$ is supported in $\left[2^{v-2} \pi, 2^{v} \pi\right]$ and $\left|\left(\frac{\partial}{\partial x}\right)^{\gamma} \hat{\eta}_{v}(x)\right|$ $\leq \tilde{A}_{\gamma} 2^{-v|\gamma|}$ for all integer $\gamma$ and $\tilde{A}_{\gamma}$ independent of $v$. Write $\hat{\eta}_{v}(x)=\sum_{n \in Z} \eta_{v}(n) e^{i n x}$. Therefore

$$
\begin{gathered}
i^{\gamma} \sum_{n \in Z} n^{\gamma} P_{v}(n)=\left.\left(\frac{d}{d x}\right)^{\gamma} \hat{P}_{v}(x)\right|_{x=0}=0 \text { for } \gamma \leq 2 M-1, \\
\left|\eta_{v}(n)\right| \leq C_{N_{1}} 2^{v}\left(1+2^{v}|n|\right)^{-N_{l}} \text { for all } N_{1} \geq 0 .
\end{gathered}
$$

Observe that $\sum_{n} \Delta^{\gamma} P_{v}(n) e^{i n x}=\left(1-e^{-i x}\right)^{\gamma} \hat{P}_{v}(x)$. Hence

$$
\begin{aligned}
\left\|\Delta^{\gamma} P_{v}(n)\right\|_{1 \infty} & \leq \int_{-\pi}^{\pi}\left|1-e^{i x}\right|^{\gamma}\left|\hat{P}_{v}(x)\right| d x \\
& \leq C 2^{2 v N} \int_{-\pi}^{\pi}\left|\sin \frac{2^{-v-1}+1}{2} x / \sin \frac{x}{2}\right|^{2 N-\gamma} d x \leq C 2^{(|\gamma|+1) v}
\end{aligned}
$$

for $\gamma \leq 2 N-2$, where $\Delta$ denotes the difference defined by $\Delta f(n)=f(n)-f(n+1)$ for every sequence $f=\{f(n)\}_{n \in Z}$ : Let $g_{v, k}(m)=\sum_{2-v k \leq n<2-v(k+1)} P_{v}(m-n) \eta_{v}(n)$. Therefore $g_{v, k}$ is supported in $\left[2^{-v}(k-2 M-N), 2^{-v}(k+2 \bar{M}+N)\right], \sum_{n \in Z} n^{\gamma} g_{v, k}(n)=0$ for $\gamma \leq 2 M-1$ and $\left\|\Delta^{\gamma} g_{v, k}\right\|_{1 \infty} \leq C_{N_{1}}(1+|k|)^{-N_{1}} 2^{(|\gamma|+1) v}$ for some large integer $N_{1}$. For $f \in F_{p}^{\alpha q}(Z)$, we write

$$
\begin{aligned}
f & =\sum_{(v, k) \in Z_{-} \times Z}\left\langle f, \varphi_{v, k}\right\rangle \psi_{v, k} \\
& =\sum_{(v, k) \in Z_{-} \times Z} t_{v, m}^{r}\left(\left(\sum_{k \in Z}\left\langle f, \varphi_{v, k}\right) 2^{-v / 2} g_{v, m-k}\left(n-2^{-v} k\right)\right) / t_{v, m}^{r}\right) \\
& =\sum_{(v, k) \in Z_{-} \times Z} t_{v, m}^{r} a_{v, m},
\end{aligned}
$$


where $t_{v, m}^{r}=\left(\sum_{k \in Z}\left|\left\langle f, \varphi_{v, k}\right)\right|^{r}(1+|k-m|)^{-2}\right)^{\frac{1}{r}}$ and $0<r<\min (p, q, 1)$. By the procedure used in $\left[5\right.$, Theorem 4.1], it suffices to prove that the $a_{v, m}$ are $(\tilde{K}, \tilde{N})$-atoms which is easy to be proved when $2 N \geq \tilde{K}+2$ and $2 M \geq \tilde{N}+1$ is chosen.

Let $\left(\phi_{v}, \psi_{v}\right) \in \mathcal{S}(Z) \times \mathcal{S}(Z)$ be an admissible pair. To prove the converse, it suffices to show that $\left\langle a_{v, k}, \phi_{v^{\prime}, k^{\prime}}\right\rangle$ is an almost diagonal matrix, i.e, there exist constants $C, \varepsilon>0$ such that

$$
\left|\left\langle a_{v, k}, \phi_{v^{\prime}, k^{\prime}}\right\rangle\right| \leq C 2^{\left(v-v^{\prime}\right) \alpha}\left(1+\frac{\left|2^{-v} k-2^{-v^{\prime}} k^{\prime}\right|}{2^{-\min \left(v, v^{\prime}\right)}}\right)^{-J-\varepsilon} \min \left[2^{\left(v-v^{\prime}\right) \frac{1+\varepsilon}{2}}, 2^{\left(v^{\prime}-v\right)\left(\frac{-1+\varepsilon}{2}+J\right)}\right]
$$

where $J=1 / \min (1, p, q)$. We prove this estimate in three cases $v=v^{\prime}, v^{\prime}\left\langle v\right.$ and $\left.v^{\prime}\right\rangle v$. Observe that $a_{v, k}(n)=\tilde{a}_{v}\left(n-2^{-v} k\right)$, where $\tilde{a}_{v}$ is an atom supported in $\left[-A 2^{-v}, A 2^{-v}\right]$, and $\phi_{v^{\prime}, k^{\prime}}(n)=2^{-v^{\prime} / 2} \phi_{v^{\prime}}\left(n-2^{-v^{\prime}} k^{\prime}\right)$. Let us denote $\hat{\tilde{a}}_{v}(\xi)=\sum_{n \in Z} \tilde{a}_{v}(n) e^{i n \xi}$. Therefore it suffices to estimate $2^{-v^{\prime} / 2} \int_{-\pi}^{\pi} \hat{\tilde{a}}_{v}(n) \phi_{v^{\prime}}(\xi) e^{i\left(2^{-v} k-2^{-v^{\prime}} k^{\prime}\right) \xi} d \xi$. By the properties of $\left\{a_{v, k}\right\}$, we have

$$
\begin{aligned}
& \left|\left(\frac{\partial}{\partial \xi}\right)^{\alpha} \tilde{\tilde{a}}_{v}(\xi)\right| \leq C 2^{-\left(|\alpha|+\frac{1}{2}\right) v} \\
& \left|\left(\frac{\partial}{\partial \xi}\right)^{\alpha} \tilde{\tilde{a}}_{v}(\xi)\right|=\left|\sum_{n} \tilde{a}_{v}(n) n^{\alpha} e^{i n \xi}\right| \\
\leq & \left|\sum_{n} \tilde{a}_{n}(n) n^{\alpha} \sum_{|\beta| \leq \bar{N}-|\alpha|}(i n \xi)^{\beta} / \beta !\right|+\sum_{n}\left|\tilde{a}_{v}(n)\right| n^{\tilde{N}+1}|\xi|^{\bar{N}-|\alpha|+1} \\
\leq & \left\{\begin{array}{lll}
C 2^{-v\left(\tilde{N}+\frac{3}{2}\right)}|\xi|^{\bar{N}-|\alpha|+1} & \text { for } \quad|\alpha| \leq \tilde{N} \\
C 2^{-v\left(|\alpha|+\frac{1}{2}\right)} & \text { for } \quad|\alpha| \geq \tilde{N}+1
\end{array}\right.
\end{aligned}
$$

and

$$
\left|\left(\frac{\partial}{\partial \xi}\right)^{\alpha} \hat{\tilde{a}}_{v}(\xi)\left(1-e^{-i \xi}\right)^{\bar{K}}\right| \leq C \sum_{n}\left|\Delta^{\bar{K}}\left(n^{\alpha} \tilde{a}_{v}(n)\right)\right| \leq C 2^{\left(\bar{N}-|\alpha|-\frac{1}{2}\right) v} .
$$

Therefore we have

$$
\left|\left\langle a_{v, k}, \phi_{v^{\prime}, k^{\prime}}\right\rangle\right| \leq \begin{cases}C\left(1+\left|k-k^{\prime}\right|\right)^{-J-\varepsilon} & \text { when } v=v^{\prime} \\ C\left(1+\left|k^{\prime}-2^{v^{\prime}-v} k\right|\right)^{-J-e} 2^{-\left(\bar{N}+\frac{1}{2}\right)\left|v-v^{\prime}\right|} & \text { when } v^{\prime}<v \\ C\left(1+\left|k-2^{v-v^{\prime}} k^{\prime}\right|\right)^{-J-e} 2^{-\left(\bar{K}-\frac{1}{2}\right)\left|v-v^{\prime}\right|} & \text { when } v<v^{\prime}\end{cases}
$$

Observe that $\tilde{N}>J-1-\alpha$ and $\tilde{K}>\alpha+1$. Therefore Theorem 3 is proved

Remark 2. Our indices $(\tilde{K}, \tilde{N})$ on smooth atoms are larger than the ones on $R$.

Now let us see two special sequence spaces: the Hardy spaces $H^{p}(Z)=F_{p}^{0,2}(Z)$ for $0<p<\infty$ and the bounded mean oscillation space $B M O(Z)=F_{\infty}^{0,2}(Z)$. Therefore $H^{p}(Z)=l^{p}(Z)$ for $1<p<\infty$ by the Littlewood-Paley theory for sequences [4, Chapter 7] and we have $\left(H^{1}(Z)\right)^{*}=B M O(Z)$ by Theorem 2. As to the characterization of $H^{1}(R)$ in $[10$, Chapter V] and $[12$, Chapter VI], we have 


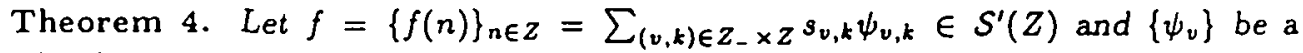
family of sequences with their Fourier series satisfying (2), (4) and (5). Then the following definitions of the Hardy space $H^{l}(Z)$ are equivalent to each other:

(i) $\sup _{F \subset Z} \times Z \sup _{\varepsilon(v, k)= \pm 1}\left\|\sum_{(v, k) \in F} \varepsilon(v, k) s_{v, k} \psi_{v, k}\right\|_{1}<\infty$, where the first supremum is taken over all finite subsets $F$ of $Z_{-} \times Z$.

(ii) $\left\|\left(\sum_{v, k}\left|s_{v, k}\right|^{2}\left|\psi_{v, k}\right|^{2}\right)^{\frac{1}{2}}\right\|_{l 1}<\infty$.

(iii) $\left\|\left(\sum_{v, k}\left|s_{v, k}\right|^{2} \bar{\chi}_{R(v, k)}^{2}\right)^{\frac{1}{2}}\right\|_{l^{1}}<\infty$ for some set $R(v, k) \subset\left[2^{-v} k, 2^{-v}(k+1)\right)$ with its measure $|R(v, k)| \geq r 2^{-v}$ for some absolutely constant $r>0$, where $2^{\frac{v}{2}} \tilde{\chi}_{R(v, k)}$ is the characteristic function of $R(v, k)$.

(iv) $\left\|\left(\sum_{v, k}\left|s_{v, k}\right|^{2} \tilde{\chi}_{u, k}^{2}\right)^{\frac{1}{2}}\right\|_{l^{1}}<\infty$, where $2^{\frac{v}{2}} \tilde{\chi}_{v, k}$ is the characteristic function of $\left[2^{-v} k\right.$, $\left.2^{-\nu}(k+1)\right)$.

(v) $f=\sum_{v, k} s_{v, k} \psi_{v, k}$ can be written as $\sum_{m} \lambda_{m} a_{m}$ with $\sum_{m}\left|\lambda_{m}\right|<\infty$, where $a_{m}=$ $\left\{a_{m}(n)\right\}_{n \in Z}$ are supported in some intervals $Q_{m}$ with $\sum_{m} a_{m}(n)=0$ and $\left\|a_{m}\right\|_{1 \infty} \leq$ $\left|Q_{m}\right|^{-1}$.

(vi) $\left\|\sup _{v \leq 0}\left|\Phi_{v} * f\right|\right\|_{l}<+\infty$, where $\left\{\Phi_{v}\right\}$ is a family of sequences such that supp $\hat{\Phi}_{v} \subset$ $\left[-2^{v} \pi, 2^{v} \pi\right], \dot{\Phi}_{v}(0)=1$. and $\left|\partial^{\alpha} \dot{\Phi}_{v}\right| \leq C_{\alpha} 2^{-\alpha v}$ for some $C_{\alpha}$ independent of $v$ and for all $\alpha \in Z_{+}$. lent:

Theorem 5. Let $b=\{b(n)\}_{n \in Z} \in S^{\prime}(Z)$. The following statements for $b$ are equiva-

(i) $b \in B M O$

(ii) $\sup _{a, b \in Z}\left(\frac{1}{b-a} \sum_{a \leq n<b}\left|b(n)-\frac{1}{b-a} \sum_{a \leq k<b} b(k)\right|^{2}\right)^{\frac{1}{2}}<\infty$.

(iii) $\sup _{v, k} 2^{v} \sum_{2-v k \leq n<2-v(k+1), 0 \geq 1 \geq v}\left|\left(\varphi_{l} * b\right)(n)\right|^{2}<+\infty$.

(iv) $\tilde{b}=\sum_{n \in Z} b(n) \chi[n, n+1) \in B M O(R)$.

The characterization of $H^{1}(Z)$ and $B M O(Z)$ by singular integral operaters will be given in Section 4.

\section{David-Journé theorem on $Z$}

We say an operator $T$ defined on $S(Z)$ is a $C$ alderon-Zygmund operator if the following hold:

(i) $T f(n)=\sum_{m} k(n, m) f(m), \quad \forall f \in S(Z)$.

(ii) $|k(m, n)| \leq C(1+|m-n|)^{-1}$.

(iii) $|k(m+1, n)-k(m, n)|+|k(m, n+1)-k(m, n)| \leq C(1+|m-n|)^{-1-\eta}$ for some $0<\eta<1$.

(iv) $T$ can be extened to a bounded operator on $l^{2}$.

We call the kernel $k=\{k(n, m)\}_{(n, m) \in Z \times Z}$ the Calderon-Zygmund kernel if it satisfies (ii) and (iii).

By the standard Calderon-Zygmund theory on $R$, we know 
Theorem 6. Let $T$ be a Calderon-Zygmund operator. Then $T$ maps $l^{P}(Z)$ into $l^{P}(Z)$ $(1<p<\infty), l^{1}(Z)$ into weak $l^{l}(Z)$ and $l^{\infty}(Z)$ into $B M O(Z)$.

Here we give the definition of $T b$ for $b \in l^{\infty}$. Let

$$
g_{k}(n)=\left\{\begin{aligned}
1 & \text { for } n=k \\
-1 & \text { for } n=0 \\
0 & \text { otherwise }
\end{aligned}\right.
$$

for $k \in Z \backslash\{0\}$. Define

$$
\left\langle T b, g_{k}\right\rangle=\left\langle T\left(b \chi_{|-2| k|, 2| k \mid\}}\right), g_{k}\right\rangle+\sum_{m} \sum_{|n| \geq 2|k|}(k(0, n)-k(m, n)) g_{k}(m) b(n) .
$$

Now we define

$$
(T b)(k)=\left\{\begin{array}{lll}
\left\langle T b, g_{k}\right\rangle & \text { for } & k \neq 0 \\
0 & \text { for } & k=0
\end{array} .\right.
$$

For example let $b(n)=\operatorname{sign} n$, the sign function, and $T$ be the Hilbert transform $H$ with the kernel $K(m, n)=\operatorname{sign}(m-n) /(m-n)$. We know $H b=+\infty$ in usual sense, but in our sense $H b(k)<+\infty$ for all $k \in Z$.

We say that $T$ has the weak boundedness property and write $T \in W B P$, if

$$
\left|\sum_{n}(T f)(n) \bar{g}(n)\right| \leq C N\left(\|f\|_{1 \infty}+N\|\Delta f\|_{1 \infty}\right)^{\frac{1}{2}}\left(\|g\|_{1 \infty}+N\|\Delta g\|_{1 \infty}\right)^{\frac{1}{2}}
$$

holds for all sequences $f, g \in l^{\infty}$ with supports contained in $\left|n-m_{0}\right| \leq N$, where $C$ is some constant independent of $f, g, N$ and $m_{0}$, and where we use the difference operator $\Delta$ as usual.

Theorem 7. Let $T$ be an operator defined on $\mathcal{S}(Z)$ with kernel satisfying (i)-(iii) Then $T$ can be extended to a bounded operator on $l^{2}$ if and only if $T \in W B P, T l \in$ $B M O(Z)$ and $T^{*} l \in B M O(Z)$. Here we use $T^{*}$ as the conjugate operator of $T$.

To prove this theorem we will use

Lemma 3. If $T \in W B P$ and $T 1=T^{*} 1=0$, then $T$ is a bounded operator on $l^{2}$.

This lemma follows from the same precedure used as in [5] and the observations that $\left\langle T \psi_{v, k}, \psi_{v^{\prime}, k^{\prime}}\right\rangle$ is an almost diagonal matrix and there is a natural map $I: f_{p}^{\alpha q}(Z) \longrightarrow$ $f_{p}^{a g}(R)$.

As to the proof of the David-Journe theorem on $R$, we want to construct a paraproduct on $Z$. Let $\left(\varphi_{v}, \psi_{v}\right) \in S(Z) \times S(Z)$ be a special admissible pair on $Z$ satisfying (2)-(5) and supp $\hat{\varphi}_{v} \subset\left\{|x| \leq \frac{9}{10} 2^{v} \pi\right\}$ for $v \leq-1$. Let $\left\{\Phi_{v}=\left\{\Phi_{v}(n)\right\}_{n \in Z}\right\}_{v \in Z_{-}}$be a family of sequences such that $\operatorname{supp} \hat{\Phi}_{v} \subset\left[-\frac{\pi}{10} 2^{v}, \frac{\pi}{10} 2^{v}\right], \hat{\Phi}_{v}(0)=1$ and $\left|D^{\alpha} \hat{\Phi}_{v}\right| \leq C_{\alpha} 2^{-\alpha v}$ for all $\alpha \in Z_{+}$and some constants $C_{\alpha}$ independent of $v$. Define the paraproduct operator $T_{b}$ by

$$
T_{b} f=\sum_{v \leq-1} \Phi_{v} *\left(\varphi_{v}(b)\left(\Phi_{v} * f\right)\right)
$$


where $\varphi_{v}(b)=\varphi_{v} * b$. Observe that the Fourier transform of $\varphi_{v}(b)\left(\Phi_{v} * f\right)$ is supported in $\left\{|x| \leq 2^{v} \pi\right\}$. Hence we can write $T_{b} f=\sum_{v, k} \phi_{v, k}\left\langle\varphi_{v, k}, b\right\rangle\left\langle f, \Phi_{v, k}\right\rangle 2^{-\frac{v}{2}}$.

Lemma 4. . Let $b \in B M O$ and $T_{b}$ be the paraproduct operator (7). Then $T_{b}$ is bounded on $l^{2}$.

Proof. Observe that supp $\hat{\psi}_{v} \cap \operatorname{supp} \hat{\Phi}_{v^{\prime}}=\emptyset$ for $\left|v-v^{\prime}\right| \geq 2$. Therefore

$$
\left\|T_{b} f\right\|_{l^{2}}^{2} \leq \sum_{k \in Z} \sum_{v \in Z_{-}}\left|\varphi_{v}(b)(k)\right|^{2}\left|\Psi_{v} * f(k)\right|^{2} .
$$

By Theorem 5 and a discrete version of Carleson measures [10, Chapter V], we have

$$
\left\|T_{b} f\right\|_{1^{2}}^{2} \leq C \sum_{k}\left(f^{*}\right)^{2}(k) \leq C \sum_{k}|M f(k)|^{2} \leq C\|f\|_{l^{2}}^{2}
$$

where

$$
\begin{aligned}
f^{*}(k) & =\sup _{\left|k^{\prime}-k\right| \leq 2^{-v}, v \in Z_{-}}\left|\Psi_{v}{ }^{*} f\left(k^{\prime}\right)\right| \\
& \leq C \sup _{\left|k^{\prime}-k\right| \leq 2^{-v}, v \in Z_{-}} \sum_{n}|f(n)|\left(1+2^{v}\left|n-k^{\prime}\right|\right)^{-2} 2^{v} \leq C M f(k) .
\end{aligned}
$$

and $M$ denotes the Hardy-Littlewood maximal operator in the above four lines (see Lemma 2 for its definition)

Now the proof of Theorem 7 reduces to proving that the kernel $K_{b}=\left\{K_{b}(m, n)\right\}_{m, n \in Z}$ of $T_{b}$ is Calderon-Zygmund kernel which is easy to. check.

\section{Stability of integer translates}

In this section, we establish a connection between a sequence $\{f(n)\} \in \mathcal{S}^{\prime}(Z)$ and its corresponding function $g(x)=\sum_{n \in Z} f(n) \phi(x-n)$, where $\phi$ is an appropriate function.

Let $\alpha \in R, J=n / \min (1, p, q), N_{0}=\max ([J-n-\alpha]+1,-1)$ and $K_{0}=[\alpha+2]_{+}$, where $[x]$ denotes the integer part of $x$ and $y_{+}=y$ for $y \geq 0$ and $y_{+}=0$ otherwise. Let $B_{l}$ be the univariate spline function defined with help of the Fourier transform by $\hat{B}_{l}(\xi)=\left(\frac{1-e^{i q}}{i \xi}\right)^{\prime}$ for $l \geq 0$.

Theorem 8. Assume $l \geq K_{0}+1, \alpha \in R, 0<p<+\infty, 0<q \leq+\infty$. For a sequerice $\{f(n)\} \in S^{\prime}(Z)$ and the function $B_{l}$, write its corresponding function $g$ as

$$
g(x)=\sum_{n \in Z} f(n) B_{l}(x-n)
$$

Then $g \in F_{p}^{\alpha q}(R)$ provided $\{f(n)\} \in F_{p}^{\alpha q}(Z)$. 


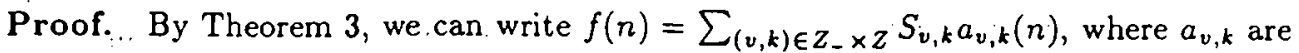
$\left(K_{0}, N_{0}\right)$ atoms on $F_{p}^{\alpha q}(Z)$. Hence $g(x)=\sum_{v, k} S_{v, k} \sum_{n} a_{v, k}(n) B_{l}(x-n)$. Now the matter reduces to showing that $\left\{\tilde{a}_{v, k}(x)\right\}=\left\{\sum_{n} a_{v, k}(n) B_{l}(x-n)\right\}$ is a family of $\left(K_{0}, N_{0}\right)$ atoms of $F_{p}^{\alpha \cdot g}(R)$. Observe that $B_{l}$ has compact support and $D^{j} B_{l}=\Delta^{j} B_{l-j}$ for $0 \leq j \leq l-2$, where $D^{j}$ and $\Delta^{j}$ denote $j$-th differention and $j$-th difference, respectively. Therefore by the properties of $\left\{a_{v, k}\right\}$ we have the following:

(i) supp $\tilde{a}_{v, k} \subset \operatorname{supp} a_{v ; k}+\operatorname{supp} B_{l} \subset\left[2^{-v}(k-l-2), 2^{-v}(k+l+2)\right]$.

(ii) $\left|D^{\gamma} \tilde{a}_{v, k}(x)\right|=\left|\sum_{n} a_{v, k}(n) \Delta^{\gamma} B_{l-\gamma}(x-n)\right|=\left|\sum_{n} \Delta^{\gamma} a_{v, k}(n) B_{l-\gamma}(x-n)\right| \leq$ $C 2^{\frac{k}{2}+\gamma v}$ for $|\gamma| \leq K_{0} \leq l-1$ and some constant $C$ independent of $v$ and $k$.

(iii) $\int x^{\gamma} \widetilde{a}_{v, k}(x) d x=\sum_{n} \sum_{s \leq \gamma} a_{v, k}(n) n^{\gamma-s}\left(\begin{array}{l}\gamma \\ s\end{array}\right) \int x^{s} B_{l}(x) d x=0 \quad$ for $|\gamma| \leq N$.

This proves that $\left\{\tilde{a}_{v, k}\right\}$ is a family of $\left(K_{0}, N_{0}\right)$ atoms of $F_{p}^{\alpha q}(R)[5$, p. 60]. Therefore $\|g\|_{F_{p}^{a q}(R)} \leq C\left\|S_{v, k}\right\|_{f_{p}^{a q}(R)} \leq C\left\|S_{v, k}\right\|_{f_{p}^{a q}(Z)} \leq C\|\{f(n)\}\|_{F_{p}^{a q}(Z)}$ and Theorem 8 holds true

Remark 3. From the proof of Theorem 8, we see that the univariate spline function $B_{l}$ can be replaced by some compactly supported function $\phi$ satisfying $D^{\gamma} \phi=\Delta^{\gamma} \phi_{\gamma}$ for some bounded compactly supported functions $\phi_{\gamma}$ and all $|\gamma| \leq K_{0}$. But the condition on $\phi$ is still too restrictive. In case $\alpha=0, q=2$ and $1<p<\infty,\|g\|_{F_{p}^{\alpha q}(R)} \leq C\|\{f(n)\}\|_{F_{p}^{\alpha q}(Z)}$ if and only if $\phi \in L^{p}(R)$ provided $\phi$ has compact support. In Theorem 13 below, we give a characterization of $\phi$ when $\alpha=0, q=2$ and $p=1$.

Now we give the reverse form of Theorem 8 .

Theorem 9. Let $\alpha \in R, 0<p<+\infty, 0<q \leq+\infty$ and $\left\{\phi_{j}\right\}_{1 \leq j \leq l}$ be some distributions with compact support. Assume that the $l \times l$ matrix $\left(\hat{\phi}_{j}\left(2 \pi k_{j^{\prime}}\right)\right)_{1 \leq j, j^{\prime} \leq l \text { has }}$ rank l for some $k, \in Z(1 \leq j \leq l)$. Write

$$
g(x)=\sum_{j=1}^{l} \sum_{n \in Z} f_{j}(n) \phi_{j}(x-n)
$$

for $f_{j}=\left\{f_{j}(n)\right\} \in \mathcal{S}^{\prime}\left(Z_{)}\right.$. Furthermore we assume

$$
\left\|\varphi_{v} * f_{j}\right\|_{1 p} \leq C_{v}<+\infty
$$

hold for every $1 \leq j \leq l, v \leq 0$ and a family of sequences $\left\{\varphi_{v} \in \mathcal{S}(Z)\right\}_{v \leq 0}$ satisfying (2),(4) and (5). Then $f_{j} \in F_{p}^{\alpha q}(Z)$ provided $e^{2 k_{j} \pi i x} g(x) \in F_{p}^{\alpha q}(R)$ for all $1 \leq j \leq l$. Furthermore

$$
\sum_{j=1}^{l}\left\|f_{j}\right\|_{F_{p}^{a q}(Z)} \leq C \sum_{j=1}^{l}\left\|e^{2 k_{j} \pi i x} g(x)\right\|_{F_{p}^{a q}(R)}
$$

holds for a constant $C$ dependent on $C_{v}$ in (8), $\left\{\varphi_{v}\right\}$ and $l$ only. 
Proof. Let $\Phi_{v}, \Phi_{v, k}$ be defined as in $[5$, p.45-46] with the modification that (2.2) in $[5]$ is replaced by supp $\hat{\Phi} \subset[-\pi, \pi] \backslash\left(-\frac{\pi}{4}, \frac{\pi}{4}\right]$ and (2.3) in [5] is replaced by $|\hat{\Phi}(\xi)| \geq C>0$ on $\left[-\frac{3}{4} \pi, \frac{3}{4} \pi\right] \backslash\left[-\frac{3}{8} \pi, \frac{3}{8} \pi\right]$. Write $e^{2 k_{j} \pi i z} \phi_{j^{\prime}}(x-n)=\sum_{(v, k) \in Z \times Z} C_{v, k}^{j, j^{\prime}}(n) \Phi_{v, k}(x)$. Therefore

$$
\begin{aligned}
C_{v, k}^{j, j^{\prime}}(n) & =\left\langle e^{2 k_{j} \pi i x} \phi_{j^{\prime}}(x-n), \Phi_{v, k}(x)\right\rangle \\
& =2^{-\frac{v}{2}} \int \hat{\phi}_{j}\left(\xi+2 \pi k_{j}\right) \overline{\hat{\Phi}}\left(2^{-v} \xi\right) e^{-i\left(n-2^{-v} k\right) \xi} d \xi
\end{aligned}
$$

Let $\tilde{\psi}_{v}^{j, j^{\prime}} \in S(Z)$ be defined by $\tilde{\bar{\psi}}_{v}^{j, j^{\prime}}(\xi)=\hat{\phi}_{j^{\prime}}\left(\xi+2 \pi k_{j}\right) \overline{\hat{\Phi}}\left(2^{-v} \xi\right)$ for $v \leq 0$. Denote $\tilde{\psi}_{v, k}^{j, j^{\prime}}(n)=2^{-v / 2} \psi_{v}^{j, j^{\prime}}\left(n-2^{-v} k\right)$. Therefore .

$$
\sum_{j^{\prime}=1}^{n} \sum_{n \in Z} f_{j^{\prime}}(n) \phi_{j^{\prime}}(x-n) e^{2 k_{j} \pi i x}=\sum_{k, v}\left(\sum_{j^{\prime}=1}^{l} \sum_{n \in Z} f_{j^{\prime}}(n) C_{v, k}^{j, j^{\prime}}(n)\right) \Phi_{\nu, k}(x)
$$

and

$$
\begin{aligned}
\left\|\left\{\sum_{j^{\prime}=1}^{l}\left\langle f_{j^{\prime}}, \tilde{\psi}_{v, k}^{j, j^{\prime}}\right\rangle\right\}_{(v, k) \in Z_{-} \times Z}\right\|_{f_{p}^{\alpha a}(Z)} & \leq\left\|\left\{\sum_{j^{\prime}=1}^{l} \sum_{n \in Z} f_{j^{\prime}}(n) C_{v, k}^{j, j^{\prime}}(n)\right\}_{(v, k) \in Z \times Z}\right\|_{f_{p}^{\circ o}(R)} \\
& \leq C\left\|e^{2 k_{j} \pi i x} g(x)\right\|_{F_{p}^{\alpha a}(R) .}
\end{aligned}
$$

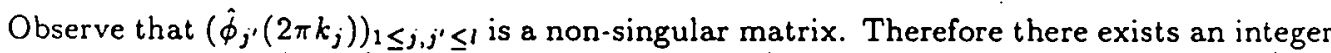
$N \geq 1$ such that $\left|\operatorname{det}\left(\hat{\phi}_{j^{\prime}}\left(\xi+2 \pi k_{j}\right)\right)\right| \geq \frac{1}{2}\left|\operatorname{det}\left(\hat{\phi}_{j^{\prime}}\left(2 \pi k_{j}\right)\right)\right|$ on $\left\{|\xi| \leq 2^{-N} \pi\right\}$, where we denote the determinant of $A$ by $\operatorname{det} A$. Denote by $\left({ }^{*} \hat{\varphi}^{j, j^{\prime}}(\xi)\right)$ the inverse matrix of $\left(\dot{\phi}_{j^{\prime}}(\xi+\right.$

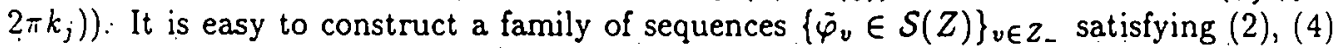
and (5) such that $\sum_{v} \sum_{j}, \hat{\tilde{\varphi}}_{v}^{j, j^{\prime \prime}}(\xi) \dot{\tilde{\psi}}_{v}^{j^{\prime}, j^{\prime \prime}}(\xi)=\delta_{j, j}$, on $\left[-2^{-N-3} \pi, 2^{-N-3} \pi\right]$, where $\hat{\tilde{\varphi}}_{v}^{j, j^{\prime}}(\xi)=$ " $\hat{\varphi}^{j} j^{\prime}(\xi) \hat{\bar{\varphi}}_{v}(\xi)$ and $\delta_{i j}$ is the Kronekker symbol. Let $\Psi \in S(Z)$ such that $\Psi(\xi)=1$ on $\left[2^{-N-4} \pi, \pi\right] \cup\left[-\pi,-2^{-N-4} \pi\right]$, and $\operatorname{supp} \hat{\Psi} \subset\left[2^{-N-5} \pi, \pi\right] \cup\left[-\pi,-2^{-N-5} \pi\right]$. Denote $F ;=$ $f_{j}-f_{j} * \Psi$. Therefore : we have.

$$
F_{j}=\sum_{j^{\prime}, j^{\prime \prime}=1}^{l} \sum_{\substack{v<-N \\ k \in Z}}\left(F_{j^{\prime}}, \tilde{\psi}_{v, k^{\prime \prime}}^{j^{\prime \prime}, j^{\prime}}\right\rangle \tilde{\varphi}_{v, k}^{j, j^{\prime \prime}}
$$

and

$$
f_{j}=\sum_{j^{\prime}, j^{\prime \prime}=1}^{1} \sum_{v<-N}\left\langle f_{j^{\prime}}, \tilde{\psi}_{v, j^{\prime \prime}}^{j^{\prime \prime}, j^{\prime}}\right\rangle \tilde{\varphi}_{v, k}^{j, j^{\prime \prime}}+\sum_{j^{\prime}=1}^{1} f_{j^{\prime}} * \Psi_{j, j^{\prime}}
$$

where supp $\hat{\Psi}_{j, j^{\prime}} \subset\left[2^{-N-5} \pi, \pi\right] \cup\left[-\pi,-2^{-N-5} \pi\right]$. Therefore 


$$
\begin{aligned}
& \left\|\left(\sum_{\nu \leq-N-7}\left(\left|\varphi_{v} * f_{j}\right| 2^{v \alpha}\right)^{q}\right)^{\frac{1}{q}}\right\|_{1 p} \\
& \leq C \sum_{j^{\prime \prime}=1}^{1}\left\|\left(\sum_{\nu \leq-N-7}\left(\left|\varphi_{v^{\prime}} *\left(\sum_{j^{\prime}=1}^{1} \sum_{v \leq-N}\left\langle f_{j^{\prime}}, \bar{\psi}_{v, k}^{j^{\prime \prime}, j^{\prime}}\right\rangle \tilde{\varphi}_{\nu, k}^{j, j^{\prime \prime}}\right)\right|^{\nu \alpha}\right)^{q}\right)^{\frac{1}{q}}\right\|_{l^{p}} \\
& \leq C \sum_{j^{\prime \prime}=1}^{l}\left\|\sum_{j^{\prime}=1}^{l} \sum_{v \in \sum_{k \in Z}}\left\langle f_{j^{\prime}}, \bar{\psi}_{v, k^{\prime \prime}}^{j^{\prime \prime}, j^{\prime}}\right\rangle \tilde{\varphi}_{v, k}^{j, j^{\prime \prime}}\right\|_{F_{p}^{a q}(Z)} \\
& \leq C \sum_{j^{\prime \prime}=1}^{l}\left\|\sum_{j^{\prime}=1}^{l}\left\langle f_{j^{\prime}}, \bar{\psi}_{v, k^{\prime}}^{j^{\prime \prime}, j^{\prime}}\right\rangle\right\|_{f_{p}^{\alpha \rho^{\prime}(Z)}} \leq C \sum_{j^{\prime \prime}=1}^{l}\left\|e^{2 k_{j \prime \prime} \pi i x} g(x)\right\|_{F_{p}^{\circ q}(R)} .
\end{aligned}
$$

On the other hand we have

$$
\left\|\left(\sum_{-N-6 \leq v \leq 0}\left(2^{v \alpha}\left|\varphi_{\nu} * f_{j}\right|\right)^{q}\right)^{\frac{1}{q}}\right\|_{l p} \leq C \sum_{-N-6 \leq \nu \leq 0}\left\|\varphi_{v} * f_{j}\right\|_{l p}<+\infty .
$$

Combining the two estimates above, we proved Theorem 9

Remark 4. The inequality $\left\|\varphi_{v} * f_{j}\right\|_{l p}<\infty$ can be replaced by the conclusion $f_{j}=$ $\{f,(n)\} \in l^{p}$ since the convolution operators $\varphi_{v}$ are bounded for all $v \leq 0$ (see also Lemma 5 below).

Now we give a simple characterization for (8) to hold.

Lemma 5 ( see [14, Section 1.5.2]). Let $0<p<\infty$ and $f \in L^{p}(R)$ with its Fourier transform having compact support. If $\phi$ is a Schwartz function, then $\phi * f \in L^{p}(R)$.

Lemma 6 (c.f. $\left[14\right.$, Section 1.3.3]). Let $0<p<\infty$ and $f \in L^{p}(R) \cap S^{\prime}(R)$ with Fourier transform $\hat{f}$ being supported in $\left[-\frac{\pi}{2}, \frac{\pi}{2}\right]$. Then there exists a. constant $C$ independent of $f$ such that

$$
C^{-1}\left(\sum_{n \in Z}|f(n)|^{p}\right)^{\frac{1}{p}} \leq\|f\|_{L^{p}} \leq C\left(\sum_{n}|f(n)|^{p}\right)^{\frac{1}{p}}
$$

Theorem 10. Let $\alpha \in R, 0<p<+\infty, 0<q \leq+\infty$ and let $\phi_{j}, g$ and $f_{j}=$ $\left\{f_{j}(n)\right\}_{n \in Z}$ be as in Theorem 9. Assume the integer translates of $\left\{\phi_{j}\right\}_{j=1}^{l}$ are globally linearly independent for tempered sequences. If $e^{2 k \pi i x} g(x) \in F_{p}^{\alpha q}(R)$ for $k \in Z$, then (8) holds where $g(x)=\sum_{j=1}^{l} \sum_{n \in Z} f_{j}(n) \phi_{j}(x-n)$.

Proof. Obviously it suffices to prove $\left\|\Phi_{x_{0}} * f_{j}\right\|_{l p}<+\infty$, for all $x_{0} \in[-\pi, \pi] \backslash 0$ and some $\Phi_{x_{0}} \in \mathcal{S}(Z)$ with $\dot{\Phi}_{x_{0}}\left(x_{0}\right) \neq 0$. For fixed $x_{0} \in[-\pi, \pi] \backslash\{0\}$, there exists $\left\{v_{j} \in Z\right\}$ such 
that the matrix $\left(\hat{\phi}_{j^{\prime}}\left(x_{0}+2 v j \pi\right)\right)$ has rank $l$ by the definition of global linear independence [11]. Since $x_{0} \neq 0$, there exists $v_{j}^{\prime}$ such that $\hat{\Phi}_{v_{j}^{\prime}}\left(x_{0}+2 v_{j} \pi\right) \neq 0$, where $\Phi_{j}$ is defined as in $[5, \mathrm{p} .45]$. Let $\tilde{\Phi}$ be a smooth function with its Fourier transform supported in $\left[x_{0}-\varepsilon, x_{0}+\varepsilon\right]$ such that

$$
\begin{aligned}
& \left|\operatorname{det} \hat{\phi}_{j^{\prime}}\left(\xi+2 v_{j} \pi\right)\right| \geq \frac{1}{2}\left|\operatorname{det} \hat{\phi}_{j^{\prime}}\left(x_{0}+2 v_{j} \pi\right)\right|>0 \\
& \left|\hat{\Phi}_{v^{\prime}}\left(\xi+2 v_{j} \pi\right)\right| \geq \frac{1}{2}\left|\hat{\Phi}_{v_{j^{\prime}}}\left(x_{0}+2 v_{j} \pi\right)\right|>0
\end{aligned}
$$

on $\left[x_{0}-2 \varepsilon, x_{0}+2 \varepsilon\right]$ and $\tilde{\tilde{\Phi}}\left(x_{0}\right)=1$, where $\varepsilon<\frac{1}{4}\left|x_{0}\right|$ is chosen small enough. Recall that $e^{2 k \pi i x} g(x) \in F_{p}^{\alpha q}(R)$. Therefore $\Phi_{v_{j}, *}\left(e^{2 v_{j} \pi i x} g(x)\right) \in L^{p}(R)$ and $\tilde{\Phi} *\left(e^{2 v_{j} \pi i x} g(x)\right) \in L^{p}(R)$

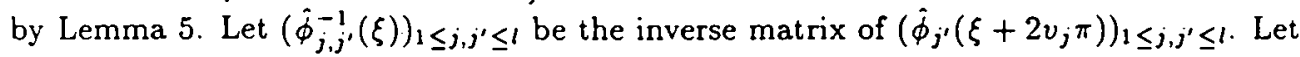

$$
\hat{\tilde{\Psi}}(\xi)=\left\{\begin{array}{ll}
1 & \text { when }\left|x_{0}-\xi\right|<\varepsilon \\
0 & \text { when }\left|x_{0}-\xi\right|>2 \varepsilon
\end{array} .\right.
$$

Denote by $\hat{\tilde{\phi}}_{j, j^{\prime}}=\hat{\tilde{\Psi}} \hat{\phi}_{j, j^{\prime}}^{-1}$ and by $\tilde{\phi}_{j, j^{\prime}}$ its inverse Fourier transform. Therefore by Lemma 5 we have $\sum_{j=1}^{l} \tilde{\phi}_{j, j^{\prime}} *\left(\tilde{\Phi} * e^{2 v_{j} \pi i x} g(x)\right) \in L^{p}(R)$. By an easy computation of Fourier transform we have

$$
\sum_{j=1}^{l} \tilde{\phi}_{j, j^{\prime}} *\left(\tilde{\Phi} * e^{2 v_{j} \pi i x} g(x)\right)=\sum_{n \in Z} f_{j^{\prime}}(n) \tilde{\Phi}(x-n) .
$$

By the sampling theorem of Lemma 6, we have $\sum_{m \in Z}\left|\sum_{n \in Z} f_{j^{\prime}}(n) \tilde{\Phi}(m-n)\right|^{p}<+\infty$. Recall that $\tilde{\tilde{\Phi}}\left(x_{0}\right) \neq 0$. Thus Theorem 10 is proved

Theorem 11. Let $\alpha \in R, 0<p<\infty$ and $0<q \leq+\infty$. Let $\left\{\phi_{j}\right\}_{j=1}^{\prime}$ be a family of compactly supported distributions so that $l \times(+\infty)$ matrix $\left(\hat{\phi}_{j}(2 k \pi)\right)_{1 \leq j \leq l, k \in Z \text { has rank }}$ l. Then the following conditions are equivalent:

(i) The integer translates of $\left\{\phi_{j}\right\}_{j=1}^{1}$ are globally linearly independent for tempered sequences.

(ii) There exists a finite subset $K \subset Z$ and a constant $C$ independent of $\left\{f_{j}\right\}_{j=1}^{l}$ such that

$$
\sum_{j=1}^{l}\left\|f_{j}\right\|_{F_{p}^{\circ}(Z)} \leq C \sum_{k \in K}\left\|e^{2 \pi k i \cdot} g(\cdot)\right\|_{F_{p}^{\circ \uparrow}(R)},
$$

where $f_{j}=\left\{f_{j}(n)\right\} \in \mathcal{S}^{\prime}(Z)$ and $g(x)=\sum_{j=1}^{l} \sum_{n \in Z} f_{j}(n) \phi_{j}(x-n)$.

Theorem 11 follows easily from Theorems 9-10 and the Fourier characterization of global linear independence by Ron [11].

Corollary. Let $1<p<\infty$ and $\left\{\phi_{j} \in L^{p}\right\}_{j=1}^{l}$ be a family of compactly supported functions. Then the following conditions are equivalent: 
(i) The integer translates of $\left\{\phi_{j}\right\}_{j=1}^{l}$ are globally linearly independent for tempered sequences.

(ii) There exists a constant $C$ independent of $\left\{f_{j}\right\}_{j=1}^{l}$ such that $\sum_{j=1}^{l}\left\|f_{j}\right\|_{l \mathrm{p}} \leq$ $C\|g\|_{L^{p}}$, where $f_{j}=\left\{f_{j}(n)\right\} \in \mathcal{S}^{\prime}(Z)$ and $g(x)=\sum_{i=1}^{l} \sum_{n \in Z} f_{j}(n) \phi_{j}(x-n)$.

The corollary follows from the observations that $\left\|e^{i x \theta} g\right\|_{L^{p}}=\|g\|_{L^{p}}$ and $\left\|\left\{e^{i n \theta} f(n)\right\}\right\|_{l^{p}}$ $=\|\{f(n)\}\|_{i p \text {. }}$

Remark 5. (i) implies (ii) when the condition $\left\{\phi_{j}\right\}_{j=1}^{l}$ in the above corollary having compactly support is replaced by $\hat{\phi}_{j} \in \mathcal{S}^{\prime}(R)$ being smooth functions for $1 \leq j \leq l$. In particular it suffices to assume that the $\hat{\phi}_{j}$ are continuous local $L^{q}$-multipliers for some $q>\max \left(p, \frac{p}{p-1}\right)$. When we assume $l=1$ in Theorem 11 , the matrix $\left(\hat{\phi}_{1}(2 k \pi)\right)$ having rank 1 can be replaced by $\hat{\phi}(0)=1$ and the set $K$ can be $\{0\}$. This fact will be used in the proof of Theorem 12 below.

Before we finish this section, we give an application of Theorem 9 to the characterization of $H^{1}(Z)$ by singular integral operators. Let the Hilbert transform $H$ on $\mathrm{Z}$ be defined by

$$
H f(n)=\sum_{m \neq n} \frac{1}{m-n} f(m) .
$$

Theorem 12. Let the Hilbert transform $H$ on $Z$ be defined by (9). Then the conclusion $f \in H^{l}(Z)$ and $f, H f \in l^{1}(Z)$ are equivalent.

Proof. The necessity follows from the atomic decomposition of $H^{1}(Z)$ and an easy reduction (see Theorem $4(v)$ ). Now we assume $f \in l^{1}$ and $H f \in l^{1}$. Let $\phi$ be a compactly supported $C^{2}$-function with $\int \phi d x=1$. For example, $\phi$ may be the univariate spline function $B_{4}$. Define a function $\tilde{f}$ on $R$ by $\tilde{f}(x)=\sum_{n \in Z} f(n) \phi(x-n)$. Obviously, $\tilde{f} \in$ $L^{1}(R)$. Let $\tilde{H}$ be the Hilbert transform on $R$ defined by

$$
\tilde{H} \tilde{f}(x)=\int \frac{1}{x-y} \tilde{f}(y) d y .
$$

Therefore for $n \leq x<n+1$ we have

$$
\begin{aligned}
\tilde{H} \tilde{f}(x) & =\sum_{|m-x| \geq 2 N+1} f(m) \int\left(\frac{1}{x-y}-\frac{1}{n-m}\right) \phi(y-m) d y \\
& +\sum_{|m-x| \geq 2 N+1} \frac{f(m)}{n-m}+\frac{1}{2} \sum_{|m-x| \leq 2 N} f(m) \int \frac{\phi(x-y-m)-\phi(x+y-m)}{y} d y
\end{aligned}
$$

and

$$
|\tilde{H} \tilde{f}(x)| \leq|H f(n)|+C \sum_{m \in Z}|f(m)| \frac{1}{1+|n-\cdot m|^{2}}
$$


where $N$ is an positive integer such that supp $\phi \subset[1-N, N-1]$ and $C$ is a constant independent of $f$. Hence $\tilde{H} \tilde{f} \in L^{1}(R)$ and $\tilde{f} \in H^{1}(R)$, the Hardy space on $R$. By Theorem 9 and the conclusion $\{f(n)\} \in l^{1}(Z)$ we get $f \in H^{1}(Z)$

Theorem 13. Let $\phi$ be a compactly supported function with $\hat{\phi}(0) \neq 0$. Then

$$
\left\|\sum_{n \in Z} f(n) \phi(x-n)\right\|_{H^{2}(R)} \leq C\|\{f(n)\}\|_{H^{1}(Z)}
$$

holds for all $f=\{f(n)\} \in H^{1}(Z)$ and a constant $C$ independent of $f$ if and only if $\phi$ and $\dot{H} \phi$ are locally integrable, where $\tilde{H}$ is the Hilbert transform on $R$ defined by (10).

Proof. Assume that $\phi$ is supported in $[-N, N]$. Define

$$
f_{0}(n)=\left\{\begin{array}{rl}
1 & n=0 \\
-1 & n=3 N \\
0 & \text { otherwise }
\end{array}\right.
$$

Therefore $\left\{f_{0}(n)\right\} \in H^{1}(Z)$ and the necessity follows from the observations that

$$
\begin{gathered}
2\|\phi\|_{L^{1}(R)}=\left\|\sum_{n \in Z} f_{0}(n) \phi(x-n)\right\|_{L^{1}(R)} \\
\int_{\frac{3 N}{2}}^{\frac{3 N}{2}}|\tilde{H} \phi(x)| d x \leq\left\|\sum_{n \in Z} f_{0}(n) \phi(x-n)\right\|_{H^{i}(R)}+C\|\phi\|_{L^{1}(R)} .
\end{gathered}
$$

On the other hand, we have $\left\|\sum_{n \in Z}^{\prime} f_{0}(n) \phi(x-n)\right\|_{L^{1}(R)} \leq \sum_{n \in Z} \mid f_{0}(n)\|\| \phi \|_{L^{1}(R)}$ and

$$
\begin{aligned}
& \int_{-\infty}^{\infty}\left|\tilde{H}\left(\sum_{n \in Z} f(n) \phi(x-n)\right)\right| d x \\
& \quad \leq \sum_{m=-\infty}^{\infty} \int_{m}^{m+1} \sum_{|n-m| \geq 2 N}|f(n)| \int\left|\frac{1}{x-n-y}-\frac{1}{m-n}\right||\phi(y)| d y \\
& \quad+\sum_{m=-\infty}^{\infty}\left|\sum_{|n-m| \geq 2 N} \frac{f(n)}{m-n}\right||\dot{\phi}(0)|+\sum_{m=-\infty}^{\infty} \sum_{|n-m| \leq 2 N}|f(n)| \int_{m}^{m+1}|\tilde{H} \phi(x-n)| d x \\
& \leq C\|\phi\|_{L^{1}(R)}\|f(n)\|_{H^{1}(Z)}+C \int_{-2 N}^{\mid N}|\tilde{H} \phi(x)| d x \sum_{n \in Z}|f(n)|<\infty .
\end{aligned}
$$

Thus Theorem 13 is proved

Also we have the following decomposition of $B M O(Z)$ by Theorems 3 and 12 .

Theorem 14. Let $H$ be the Hilbert transform on $Z$ defined by (9). Then $b \in B M O(Z)$ if and only if there exist $b_{1}$ and $b_{2} \in l^{\infty}$ such that $b=b_{1}+H b_{2}$. 
Remark 6. From the proof of Theorem 12 we get that if the identity operator I and Calderon-Zygmund operator $T$ characterize the Hardy space $H^{1}(R)$, then the identity operator $I$ on $Z$ and the operator $T^{\prime}$ with kernel being the restriction of that of $T$ to $Z \times Z \backslash\{(m, m), m \in Z\}$ characterize $H^{1}(Z)$.

\section{REFERENCES}

[1] Cohen, A. and Q. Sun: An arithmetic characterization of the conjugate quadrature filters associated to orthonormal wavelet bases. SIAM J. Math. Anal. (to appear).

(2) Daubechies, I.: Orthonormal bases of compactly supported wavelets. Comm. Pure Appl. Math. 41 (1988), $909-996$.

(3) David, G. and J. L. Journè: A boundedness criterion for generalized Calderon-Zygmund operators. Ann. Math. 120 (1984), $371-397$.

(4) Edwards, R. E. and G. I. Gaudry: Littlewood-Paley and Multiplier Theory. New York: Springer-Verlag 1977.

(5) Frazier, M. and B. Jawerth: A discrete transform and decomposition of distribution space. J. Funct. Anal. 93 (1990), $34-170$.

[6] Frazier, M. and R. Torres: The sampling theorem, $\varphi$-transform and Shannon wavelets for $R, Z, T$ and $Z_{N}$. In: Wavelets: Mathematics and Applications (eds: Benedetto and M. Frazier). CRC Press (to appear)

(7) Jia, R-Q and C. A. Micchelli: Using the refinement equations for the construction of pre-wavelet II: power of two. In: Curves and Surfaces (eds.: P. J. Laurent, A. Le Méhauté and L. L. Schumaker). New York: Academic Press 1990.

(8) Jia, R-Q, J-Z Wang: Orthogonality and Stability associated with wavelet decomposition. Proc. Amer Math. Soc. (to appear).

(9) Hardy, G., Littlewood, J. E. and G. Polya: Inequalities, 2nd edition. Cambridge: Cambridge Univ. Press 1952

(10) Meyer, Y.: Ondelettes et Opérateurs I, II, III. Paris: Herman 1990.

[11] Ron, A.: A necessary and sufficient condition for the linear independence of the integer translates of a compactly supported distribution. Constr. Approx. 5 (1989), 297 - 308.

(12) Strömberg, J.O. and A. Torchinsky: Weighted Hardy Spaces (Lecture Notes in Math.: Vol. 1381). Berlin: Springer-Verlag 1989.

(13) Torres, R.: Spaces of sequences, sampling theorem and functions of exponential type. Studia Math. $100(1990), 51-74$.

[14] Triebel, H.: Theory of Function Spaces. Leipzig: Akad. Verlagsges., 1983. 